\title{
Rapid capabilities generation and prompt effects in offensive cyber operations
}

\section{JD Work}

Marine Corps University and Columbia University; Quantico, $\mathrm{VA}^{1}$

Technology and The Cyber Domain: Implications for Intelligence. International Studies

Association Annual Conference. Las Vegas. April 2021.

\begin{abstract}
Current framing of "cyber weapons" employment has struggled to conceptualize the character and role of offensive cyber capabilities - faltering due to technical complexities and changing interactions between vulnerabilities, attack surfaces, implants, targets and hardening measures within an evolving ecosystem. Over time, however, certain key dynamics of offensive capabilities employment have become accepted within the literature. These include the recognition of tradeoffs between stealth and persistence, between nondetection and effects, capabilities variation over time based on disclosure and differing system configurations, as well as proliferation potential of capabilities once employed. Recent inquiry has considered level of effort and planning horizon required to acquire accesses and tailor capabilities for complex or sustained effects at operational and strategic levels - challenging assumptions underpinning "bolt out of the blue" engagement scenarios, including "Electronic Pearl Harbor". Yet while acknowledgement of operational constraints is welcomed, recent discourse risks overstating the temporal and resource investment dimensions involved in offensive cyber options. We explore understudied factors enabling potential rapid capabilities generation across multiple offensive program types, and consider outcomes from prompt effects delivered under several scenarios. We further consider implications for warning intelligence, countering strategies including persistent engagement, and strategic (in)stability.
\end{abstract}

\section{Introduction}

The manner in which offensive cyber operations may threaten the state remains a subject of much uncertainty. As major cyber incidents continue to dominate headlines in both recurring crisis events and routine criminal matters, there remains substantial barriers to policymaker (and scholarly) understanding the potential option space of engagements. These barriers arise due to opacity and secrecy which characterizes that domain, as well as the continuing immaturity around concepts of operation as commonly described in a frequently changing technology ecosystem. The result has been a pendulum swing from fears of bolt from the blue attack, to a better recognition of longer-term campaign dynamics involved in persistent intrusion and operational preparation of the environment for tailored destructive effects involving critical infrastructure targets. Yet this swing has elided recent developments that present hostile military and intelligence service with options to rapidly generate new offensive capabilities to hold at risk substantial economic equities. Such options, particularly within a

\footnotetext{
${ }^{1}$ The views and opinions expressed here are those of the author, and do not necessarily reflect the official policy or position of any agency of the US government or other organization.
} 
crisis context, build upon illicit markets already leveraged for sustained criminal access now most frequently monetized through extortion, but which adversary planners and operators may seek to turn to new strategic ends.

This paper consists of four parts. In the first section, we review the evolution of different concepts of offensive cyber capabilities as access and effects options, as this understanding of cyberweapons shifted from considerations of immediate impact (in analogy to strategic airstrike or nuclear delivery, based on specific technical features of early vulnerabilities exploited for offensive purpose) - towards recognition of the character of extended campaigns required to achieve punishment and coercive objectives against contemporary systems and networks. In the second section, we consider the mechanisms by which hostile military and intelligence services may leverage the extensive underground markets supporting continuing criminal enterprises engaged in ransomware extortion to bootstrap capabilities for new offensive operations within a short timeline during a crisis event. In the third section, we evaluate the quality of potential access and impact of likely destructive events in a cold start scenario, through survey of 949 initial access brokerage and ransomware incidents in Europe, the United States, and Canada reported over the course of a yearlong period, in order to bound potential scope and severity of this concept of operations. We find that in any given 30 day window, approximately one hundred typically mid-sized enterprise targets across strategic critical infrastructure sectors may be routinely held at risk for disruptive and destructive effects (with potential to further extend reach through multiple factors compressing targeting timelines), and provided broad estimates of aggregate economic activity across these enterprises.

We conclude that rapid capabilities generation approaches leveraging criminal capabilities may indeed provide viable options for short, sharp campaigns delivering prompt effects from a cold start scenario. However, it remains an untested proposition that such effects can reach a true strategic threshold, given that the observed incidents already represent current burden on Western economies, although a very different political response to deliberately concentrated destructive actions accompanied by specific messaging may well be expected than when facing routine criminal opportunism. We note that such a potential scenario poses serious challenges for warning intelligence, which must distinguish between observables of routine criminal profit and subtle shifts in the same activity for strategic purpose. Considering these intrusions in aggregate as a type of continuously ongoing operational preparation of the environment, resulting in pre-positioned access to deploy destructive effects, highlights the destabilizing nature of these capabilities options. These findings also highlight the latent strategic value of permitted, or even encouraged or supported, high optempo cyber crime as an independent capability reserve for unprincipled state services. Findings also appear to validate strategic thinking which de-emphasizes prospects for deterrence and focuses instead on persistent engagement to contest adversary actions that hold US and allied interests at risk - explaining in part recent counter-cyber operations engagements such as those observed during the 2020 US Federal elections. The prospect of future such engagements, as this strategic concept proliferates to multiple actors, further extends beyond the original Russian criminal marketplaces from which these capabilities originally arose and into new conflicts. 


\section{Conceptions of offensive capabilities and their employment}

The existing literature reflects the serious challenges encountered in describing and explaining warfighting in the cyber domain to date, at any level of conflict. These issues start with the understanding of offensive cyber capabilities themselves - where it remains in dispute to even commonly refer to such offensive tooling as a "cyber weapon". ${ }^{2}$ Yet a weapon-like role for certain offensive capabilities remains undoubtably a part of design and operational use. Early conceptions about these capabilities however conflated the functions of access and effect, and assumed in part that simply exploiting a vulnerable system would be sufficient to achieve decisive impact on the target. In part, these assumptions arose out of certain exploits of common in this formative period - where early cyber attack techniques such as the "Ping of Death" and other TCP fragmentation attacks provided some technical basis for the belief that the complexities of compromise and action on objective could be considered in abstracted aggregate. ${ }^{3}$ (It remains painfully ironic that nearly 25 years later, similar exploitation leveraging malformed packets delivered against vulnerable IPv6 stacks once again briefly resurfaced as a contemporary concern. $)^{4}$ Likewise, rapid propagation of malware through exploitation of wormable vulnerabilities also reinforced the idea that any cyberweapon could be expected achieve rapid, decisive impact at scale - as demonstrated in the early Morris, Nimda, Code Red, Slammer, Witty, and similar cases of the period. ${ }^{5}$

\footnotetext{
${ }^{2}$ Dale Peterson. "Offensive Cyber Weapons: Construction, Development, and Employment", Journal of Strategic Studies. 2013. ; Dusan Repel and Steven Hersee. "The Ingredients of Cyber Weapons". 10th International Conference on Cyber Warfare and Security. Kruger National Park, South Africa. 24-25 March 2015.; Robert E. Schmidle Jr., Michael Sulmeyer, Ben Buchanan. "Nonlethal Weapons and Cyber Capabilities". In George Perkovich, Ariel E. Levite [eds] Understanding Cyber Conflict: Fourteen Analogies. Washington, DC: Georgetown University Press. 2017; Jeffrey T. Biller and Michael N. Schmitt. "Classification of Cyber Capabilities and Operations as Weapons, Means, or Methods of Warfare." International Law Studies. Vol 95. 179-225. 2019. ${ }^{3}$ Ed M. Kenney. Ping o' death. http://www.insecure.org/sploits/ping-o-death.html, 1996.

${ }^{4}$ Duo. "Microsoft Fixes Ping Of Death Flaw In Windows". 13 October 2020.; McAfee. "CVE-2020-16898: "Bad Neighbor". 13 October 2020.; Rapid7. "There Goes the Neighborhood: Dealing With CVE-2020-16898 (and CVE2020-1656) (aka 'Bad Neighbor')". 13 October 2020.

${ }^{5}$ Hilarie Orman. "The Morris worm: A fifteen-year perspective." IEEE Security \& Privacy 1, no. 5 (2003): 35-43. ; Steven Furnell, Eugene H. Spafford. "The Morris Worm at 30." ITNOW 61, no. 1 (2019): 32-33. ; Andrew Machie, Jenssen Roculan, Ryan Russell, and M. V. Velzen. "Nimda worm analysis." SecurityFocus, 2001.; David Moore, Colleen Shannon, and K. Claffy. "Code-Red: A Case Study on the Spread and Victims of an Internet Worm." Proceedings of the 2nd ACM SIGCOMM Workshop on Internet measurement (IMW). Marseille France. November 2002.; Peter G. Capek, David M. Chess, Steve R. White, and Alan Fedeli. "Merry Christma: An Early Network Worm." IEEE Security \& Privacy 1, no. 5 (Sept.-Oct. 2003): 26-34. ; Nicholas Weaver, Vern Paxson, Stuart Staniford, and Robert Cunningham. "A Taxonomy of Computer Worms," WORM '03: Proceedings of the 2003 ACM Workshop on Rapid Malcode, Washington, DC, USA, 27 October 2003; Symantec. "W32.Welchia.Worm." 18 August 2003. ; David Moore, Vern Paxson, Stefan Savage, Colleen Shannon, Stuart Staniford, and Nicholas Weaver. "Inside the slammer worm." IEEE Security \& Privacy 1, no. 4 (2003): 33-39. ; Colleen Shannon, and David Moore. "The spread of the witty worm." IEEE Security \& Privacy 2, no. 4 (2004): 46-50.; Dwayne Edwards. "Netsky.p Mass Mailer Worm Analysis." SANS Institute. 9 January 2005.
} 
These concepts resulted in recurring fears of that offensive cyber actions would play out as a potential "bolt from the blue", including devasting surprise attack analogized to an "Electronic Pearl Harbor". ${ }^{6}$ This concept would continue to be repeated for nearly three decades at increasing levels of seniority, but as time progressed a more mature understanding of offensive operations would cause many to question the continuing utility of the construct. ${ }^{7}$ Rather, scholars and analysts came to recognize what planners and operators had learned in practicethat sustained intrusion required extensive time and effort to obtain initial footholds in high value networks, move laterally for maximum potential utility across a relevant number of ley nodes, and tailor effects payloads for heterogenous and sometimes unique operational technology environments. To understand these actions it is best to consider the unit of analysis not as the individual incident but rather the campaign. ${ }^{8}$ These campaigns could stretch weeks, months or even years; and did not guarantee lasting impact on the targeted objective. Operators could prioritize their decisions regarding access and effect according to different postures, and had to remain mindful that vulnerabilities in the targeted systems were not absolute conditions but rather had to be weighed according to a shifting series of factors for which different optimization strategies might produce very different outcomes. ${ }^{9}$

The recognition of these dynamics has led planners to think very differently about how to husband such capabilities in routine competition and conflict below the threshold of armed conflict, while preserving options in the arsenal for crisis and higher order conflict scenarios including wartime use. This segmentation of routine operations from "break glass" reserved capabilities is of course not absolute, as vulnerability rediscovery collisions and the natural software development lifecycle shortens the expected lifespan of viable Oday exploits. ${ }^{10}$ Likewise, the relative offensive utility of any given reserved capability must be weighted against

\footnotetext{
${ }^{6}$ Winn Schwartau. Testimony before the Subcommittee on Technology and Competitiveness, Committee On Science, Space, And Technology U.S. House Of Representatives. 27 June 1991.; Emily O. Goldman, John Surdu, \& Michael Warner. "The Cyber Pearl Harbor Analogy: An Attacker's Perspective". In Emily O. Goldman and John Arquilla [eds]. Cyber analogies. Naval Postgraduate School. Monterey, 2014. ; James J. Wirtz, "The Cyber Pearl Harbor." Intelligence and National Security 32, no. 6 : 758-767. 2017.

${ }^{7}$ George Smith. "An Electronic Pearl Harbor? Not Likely." Issues in Science and Technology 15, no. 1 :68-73. 1998. ; John Arquilla. "Panetta's Wrong about a Cyber Pearl Harbor," Foreign Policy. 20 November 2012.; Lawson, Sean, and Michael K. Middleton. "Cyber Pearl Harbor: Analogy, fear, and the framing of cyber security threats in the United States, 1991-2016." First Monday. 2019.; James J. Wirtz. "The Cyber Pearl Harbor redux: helpful analogy or cyber hype?." Intelligence and National Security 33, no. 5: 771-773. 2018.

${ }^{8}$ Richard J. Harknett, Max Smeets. "Cyber campaigns and strategic outcomes." Journal of Strategic Studies 1-34. 2020

${ }^{9}$ Max Smeets. "A Matter of Time: On the Transitory Nature of Cyberweapons." Journal of Strategic Studies 41, no. 1-2 (2018): 6-32.; JD Work. "Calculating the Fast Equations: Arsenal Management Considerations in Sustained Offensive Cyber Operations." Cyber Security Project, Belfer Center for Science and International Affairs, Harvard University, 2019.; Max Smeets, JD Work. "Operational Decision-Making for Cyber Operations: In Search of a Model." Cyber Defense Review. Winter 2020: 99-116.

10 Jason Healey. "The U.S. Government and Zero-Day Vulnerabilities: From Pre-Heartbleed to Shadow Brokers". Journal of International Affairs. November 2016.; Trey Herr, Bruce Schneier, Christopher Morris. "Taking stock: Estimating vulnerability rediscovery." Belfer Cyber Security Project, Harvard University. 2017. ; Tristan Caulfield, Christos Ioannidis, David Pym. "The US vulnerabilities equities process: An economic perspective." In International Conference on Decision and Game Theory for Security, pp. 131-150. (Cham: Springer) 2017. ; Lillian Ablon, Andy Bogart. Zero days, thousands of nights: The life and times of zero-day vulnerabilities and their exploits. Rand. 2017.
} 
the defensive value across a state's critical infrastructure (and that of its key allies and economic partners), resulting in difficult decisions around the decision and timing of protective disclosures under different Vulnerability Equities Process (VEP) structures. ${ }^{11}$

However, these capabilities may also be seen differently depending on differing objectives. Adversary access, and capabilities, to deliver effects against operational technology in military and critical infrastructure contexts requires typically longer development cycles, against systems and network targets that change more slowly than commercial enterprise information technologies. Specific implants also require greater tailoring to unique features of specific targets, and the operational workflows of the organizations within which these targets exist. These features may be particularly seen in the CRASHOVERRIDE / Industroyer and TRISIS / TRITON / HATMAN industrial control systems focused malware payloads, attributed by industry to Russian intelligence services development. ${ }^{12}$ Given the need for such tailoring, it follows such options must be more carefully husbanded against need. This drives a requirement to identify alternatives that may be used with fewer constraints, without risking highly specialized options against high value targets. The widespread proliferation of multiple commodity malware variants in common use by continuing criminal enterprises points to a potential resolution of this dilemma for hostile state military and intelligence services.

\section{Underground market acquisition strategies for cold start access and effects capabilities}

Capabilities sourced from criminal underground markets may be seen as providing a latent reserve by which adversary actors may bootstrap new offensive lines of effort on an accelerated timeline, in comparison to organic development and access operations approaches. Such "cold start" scenarios may significantly reduce assumptions regarding time required for mobilization in order to deliver effects in a crisis.

Multiple state intelligence services have long recognized the value of leveraging existing capabilities developed by continuing criminal enterprises, and repurposing these capabilities for access and effects against their own targets (or for their own objectives where targeting interests overlap). Russian attributed intrusion sets were among the earliest and most prominent examples of these dynamics. These included multiple efforts leveraging commodity malware for national level campaigns, as seen in early repurposing of Zeus variants and ultimately in the long term relationship alleged to have developed with key leadership of the

\footnotetext{
${ }^{11}$ Office of Director of National Intelligence. "Commercial and Government Information Technology and Industrial Control Product or System Vulnerabilities Equity Policy and Processes. 16 February 2010." Declassified.

${ }^{12}$ Dragos. "CRASHOVERRIDE: Analyzing the Malware that Attacks Power Grids". June 2017. ; ESET. "Industroyer: Biggest threat to industrial control systems since Stuxnet". 12 June 2017. ; Dragos. "TRISIS Malware Analysis of Safety System Targeted Malware". December 2017. ; FireEye. "TRITON Attribution: Russian Government-Owned Lab Most Likely Built Custom Intrusion Tools for TRITON Attackers". 23 October 2018. ; Dragos. CRASHOVERRIDE: Reassessing the 2016 Ukraine Electric Power Event as a Protection-Focused Attack. 15 August 2019.
} 
GameOver Zeus crimeware-as-a-service enterprise. ${ }^{13}$ During the conflict with Georgia, criminal pay-per-install services provided a significant enough contribution that inventories of previously compromised drones - typically resold for other purposes such as spam botnet development were reported to have been substantially emptied at the height of the crisis. ${ }^{14}$

Notable links between different ransomware groups and the commodity botnet activities that support these actions have been described by various commercial threat intelligence sources, including complex relationships between different groups and their various tooling variants - as in the example of Ryuk ransomware operations leveraging Emotet and Trickbot access options. ${ }^{15}$ Rather than merely opportunistic sales, or infections of the same victim for the second, third, or $n^{\text {th }}$ time as declining value is progressively extracted through different criminal schemes, a higher quality of access is refined through additional effort invested in victim characterization, network reconnaissance, and lateral movement to advantageous positions within an enterprise network.

Contemporary access brokerage has also further evolved into a complex underground market segment of its own. While botnet inventory continues be resold where actors perceive value in the transaction, other actors may specialize in exploitation of different types of vulnerabilities to enable intrusion against public facing elements of an enterprise network, such as in the recent uptick in high severity router and VPN device bugs, or in recurring remote access vulnerabilities involving RDP or specific vendor solutions. ${ }^{16}$ These initial intrusion footholds are offered on the market either at fixed price, in auction, or more rarely in return for a percentage of criminal profit monetized from the victim. ${ }^{17}$ Access brokers that can deliver a steady supply of prospective new targets for later extortion are more likely to form established relationships over time with partners who can reliably deliver on the complex series of steps of hold a network hostage, execute negotiations with victims under pressure, and manage high value financial transactions not merely through cryptocurrency but also through re-integration into the traditional banking system. The offers observed in underground marketplaces therefore represent freelancers who have not developed such stable relationships, choose not to limit their partnerships, or for whatever reason may find that specific victims are not suitable for the operational boundaries or operator preferences of a reliable partner and therefore more readily disposed of via the markets. Some percentage of these offers may never find a buyer,

\footnotetext{
13 Thomas Brewster. "FBI 'Most Wanted' Cybercrime Kingpin Linked To Russian Espionage On US Government". Forbes. 5 August 2015.; Michael Schwirtz and Joseph Goldstein. "Russian Espionage Piggybacks on a Cybercriminal's Hacking". New York Times. 12 March 2017.

14 iSIGHT Partners. "Assessing the Russian / Georgian Network Conflict". 24 September 2009.

${ }^{15}$ Duo. "The Unholy Alliance Of Emotet, Trickbot And The Ryuk Ransomware". 11 January 2019.; CyberReason. "A One-two Punch of Emotet, TrickBot, \& Ryuk Stealing \& Ransoming Data". 2 April 2019. ; Intel 471. "Understanding the relationship between Emotet, Ryuk and TrickBot". 14 April 2020.

${ }^{16}$ ZScaler. "Remote Access VPNs Have Ransomware on Their Hands". 7 January 2020. ; Cyber and Infrastructure Security Agency. "Continued Exploitation of Pulse Secure VPN Vulnerability". 15 April 2020. ; eSentire.

"Ransomware Groups Exploit Remote Access Services". 17 September 2020. ; Recorded Future. "Threat actors start attacking F5 devices using recent vulnerability". 19 March 2021.

17 Digital Shadows. "Initial Access Brokers: An Excess of Access." 17 February 2021. ; Recorded Future. "Initial Access Brokers: A Deep Dive". 23 February 2021.
} 
and others may be acquired by a buyer who is unable to translate this access into effective extortion.

Established relationships built over time also likely may be leveraged at speed, and with lower probability of friction, if and when a state military or intelligence service chooses to call upon these capabilities. Some services have reportedly recruited their operators from former black hat hackers. ${ }^{18}$ Others may tolerate moonlighting or other transactional interactions even outside of strict operational necessity. ${ }^{19}$ These operators will tend to have unique real world experiences that cannot readily be replicated in sterile training environments, especially for states that have made only limited investments in their offensive program talent. Industry analysts have stated that "cybercrime is a great place to learn your trade as a future state sponsored attacker", an assessment that almost certainly holds true in a number of documented programs. ${ }^{20}$

These relationships take on even greater significance when one considers the potential for earlier use in operational test and evaluation through "live fire" events, intended to test capabilities and concepts of operation (CONOP). Such events often become an operational necessity due to lack of investment in controlled range environments for testing, or other programmatic immaturity. ${ }^{21}$ Indeed, previously observed intrusion operations against seemingly arbitrary targets have even been theorized to be part of live fire "final exam" or other key certification milestones before graduation from certain offensive training programs conducted by state services. ${ }^{22}$

These are not merely theoretical concerns, as multiple suspected incidents involving both Russian, Chinese, and otherwise unattributed operators have been proposed as potential have been live fire test events. In advance of the June 2017 NotPetya / Nyeta destructive campaign, the SANDWORM / VOODOO BEAR / IRON VIKING intrusion set deployed an earlier variant of modified commodity ransomware in a smaller scale test during December 2016 against an Eastern European target. Unlike the more widely known global incident, the test operation did not result in uncontrolled proliferation beyond the initial target network, as the Moonraker Petya demonstrator variant used here had not been adapted for lateral movement using later introduced Oday exploit features seen in subsequent operational use. ${ }^{23}$ This activity group has

\footnotetext{
${ }^{18}$ Andrew E. Kramer. "How Russia Recruited Elite Hackers for Its Cyberwar". New York Times. 29 December 2016.

${ }^{19}$ FireEye. "APT41: A Dual Espionage and Cyber Crime Operation". 7 August 2019.; Steve Stecklow, Alexandra Harney. "Exclusive: Malware broker behind U.S. hacks is now teaching computer skills in China." Reuters. 24 December 2019.

${ }^{20}$ Patrick Gray and Adam Boileau. "Exchange Oday party time for Chinese APT crew". Risky Business podcast. 3 March 2021.

${ }^{21}$ JD Work. "Who Hath Measured the (Proving) Ground: Variation in Offensive Capabilities Test and Evaluation." 15th International Conference on Cyber Warfare and Security. Old Dominion University, Norfolk, VA. March 2020.

22 Discussion under Chatham House rule. Workshop on Measuring Cyber Deterrence, Columbia University. 25 February 2019.

${ }^{23}$ ESET. "GREYENERGY: A successor to BlackEnergy". October 2018.
} 
been attributed by the US government to the Russian (Main) Intelligence Directorate (commonly still known as GRU, Гла́вное разве́дывательное управле́ние) Unit $74455 .{ }^{24}$

This pattern has likely been repeated for live fire validation of capabilities targeting operational technology environments, notably in the thus far formally unattributed case of LockerGaga ransomware infection, which victimized a prominent Norwegian integrated metals production firm in March 2019. In this incident, commodity malware was deployed mimicking certain characteristics of contemporaneous criminal ransomware extortion against both French and US firms previously attributed to FIN6 activity group, but that here differed in key operational tradecraft from these earlier operations - which raised theories of possible state nexus consistent with live fire testing. ${ }^{25}$ The observed malware variant was itself modified from earlier versions seen in continuing criminal enterprise use.

Further ransomware deployment against energy sector targets in Taiwan leveraging the COLDLOCK malware variant was observed during May 2020. This ransomware also appears to have been developed from earlier commodity malware in circulation since 2016, including the Hidden Tear, EDA2 and Freezing code families. The incidents were attributed by industry intelligence and the government of Taiwan to contractors associated with the Chinese Ministry of State Security as part of operations within the APT41 / WICKED PANDA / BARIUM intrusion set, sometimes known as WINNTI. ${ }^{26}$ While no independent international confirmation of this attribution has been publicly acknowledged, Taiwanese intelligence capabilities were likely uniquely postured to collect against this campaign. However, frequent overboard clustering of threat activity under the diffusely characterized WINNTI umbrella does suggest some caution. ${ }^{27}$ Disruptive effects from the attack resulted in impact to oil and liquified natural gas operations at the country's ports, and would likely have provided reflections of otherwise impossible to estimate dynamics of incident response, network recovery, and operational contingency actions - all key measures of target resiliency critical in understanding the potential military or strategic coercive utility of reversible effects in offensive cyber operations at scale in the event of a cross-Straits crisis.

Recent cases of ransomware delivery against multiple targets by an intrusion set attributed by industry to Iran may be also be considered as potentially precursors to such a strategy. ${ }^{28}$ Here however, Iranian capabilities appear to have been leveraged aggressively as the "best available" offensive cyber option following ongoing exchanges targeting Israeli critical infrastructure, and in which unknown operators delivered disruptive effects against Iranian port networks in

\footnotetext{
${ }^{24}$ Department of Justice. "Six Russian GRU Officers Charged in Connection with Worldwide Deployment of Destructive Malware and Other Disruptive Actions in Cyberspace." 19 October 2020.

25 Joe Slowik. "Spyware Stealer Locker Wiper: LockerGaga revisited." March 2020.

${ }^{26}$ Sean Lyngaas. "Taiwan's state-owned energy company suffers ransomware attack". Cyberscoop. 5 May 2020.; Trend Micro. "Targeted Ransomware Attack Hits Taiwan Organizations". 6 May 2020. ; Taipei Times. "Bureau names ransomware culprits". 17 May 2020.

${ }^{27}$ Chronicle. "Winnti: More than just Windows and Gates". 15 May 2019.

${ }^{28}$ ClearSky. "Operation Quicksand". 15 October 2020. ; Checkpoint. "Ransomware Alert: Pay2Key." 6 November 2020. ; Checkpoint. "Pay2Key - The Plot Thickens". 12 November 2020.
} 
apparent response. ${ }^{29}$ Assessment of these cases is further complicated by earlier identified incidents for apparent criminal profit motivations involving the SamSam malware variant by Iranian nationals operating from within Iran, tracked by industry as BOSS SPIDER / GOLD LOWELL. ${ }^{30}$ The indicted ransomware operators involved in these extortion incidents were found to have extensive prior interactions with Iranian Revolutionary Guards Corps elements, including prior suspected involvement in offensive operations for state-nexus objectives. ${ }^{31}$

\section{Evaluating viable access options at arbitrary moments in time}

In order to understand contemporary options available to state military and intelligence services that may wish to pursue a cold start access concept of operation in order to generate prompt offensive effects against strategic targets, a new dataset was developed. This dataset relied upon surveys of underground market transactions observed by the industry security research community, including victims advertised by initial access brokers, and victims identified in extortion demands by ransomware continuing criminal enterprise operators. The dataset encompasses 949 incidents impacting entities in the United and Europe between February 2020 and February 2021. This timeframe was selected specifically to encompass the period of globally acknowledged impact of the COVID-19 pandemic, deliberately to exclude confounding factors introduced by discontinuities between operations under prior order and those now taking place in a radically changed political and business environment. Cases were further selected only from established brokers and ransomware operators with extensive, validated campaign records tracked broadly by multiple researchers. These included AKO / MedusaLocker / RanzyLocker, Avaddon, Babuk, Conti / Ryuk, Clop, Cuba, Darkside, Dopplepaymer / DOPPEL SPIDER, Egregor, Everest / Everbe 2.0, Lockbit, Maze / TWISTED SPIDER, Mountlocker, Netwalker / CIRCUS SPIDER, Nefilim, Pay2Key, Pysa / Mespinoza, Ranomexx, RagnarLocker / VIKING SPIDER, REvil / Sodnobicki / PINCHY SPIDER, Sekhmet, and Suncrypt malware variants. ${ }^{32}$

\footnotetext{
${ }^{29}$ JD Work and Richard Harknett. "Troubled vision: Understanding recent Israeli-Iranian offensive cyber exchanges." Cyber Statecraft Initiative, Atlantic Council. 22 July 2020.

${ }^{30}$ US Department of Justice. "Two Iranian Men Indicted for Deploying Ransomware to Extort Hospitals, Municipalities, and Public Institutions, Causing Over $\$ 30$ Million in Losses." 28 November 2018. ; US Department of the Treasury. "Treasury Designates Iran-Based Financial Facilitators of Malicious Cyber Activity and for the First Time Identifies Associated Digital Currency Addresses". 28 November 2018.; Secureworks. "SamSam Ransomware Campaigns". 15 February 2018. ; CrowdStrike. "An In-Depth Analysis of Samsam Ransomware and BOSS SPIDER". 21 May 2018. ; Sophos. "SamSam: The (Almost) Six Million Dollar Ransomware". 18 July 2018.

${ }^{31}$ Charlie Cullen. "The Men Who Never Were: Assessing Ties Between the Samsam Ransomware Campaign and the IRGC". BSides Atlanta. 28 March 2020.

32 SentinelOne. "How MedusaLocker Ransomware Aggressively Targets Remote Hosts". 29 November 2019. ; Cisco TALOS. "Threat Spotlight: MedusaLocker". 23 April 2020.; SentinelLabs. "Ranzy Ransomware: Better Encryption Among New Features of ThunderX Derivative". 18 November 2020.; Cofense. "Avaddon Ransomware Joins Data Exfiltration Trend".25 August 2020. ; Javier Yuste, Sergio Pastrana. "Avaddon ransomware: an in-depth analysis and decryption of infected systems". Preprint. February 2021. arXiv:2102.04796 [cs.CR]; Sophos. "Conti ransomware: Evasive by nature". 16 February 2021. ; VMWare Carbon Black. "TAU Threat Discovery: Conti Ransomware". 8 July 2020. ; Catalin Cimpanu. "Conti (Ryuk) joins the ranks of ransomware gangs operating data leak sites". ZDNet. 25 August 2020; Cyberint. "Babuk Locker". 29 January 2021. McAfee. "Technical Analysis of Babuk Ransomware". 23
} 
Ransomware operators identify victims in deliberate leak disclosures through publicity sites intended to place additional coercive pressure on compromised firms to pay the criminal demands or face further release of data stolen from the target's network (which is then locally encrypted), rather than merely execute business recovery contingency plans in order to restore from backup. ${ }^{33}$ While these victims are often subsequently named in public press reporting and by security researchers, all identity information is omitted from this dataset to prevent compounding victimization of the targeted entities. Targets are instead characterized only by critical infrastructure sector, as defined by Presidential Policy Directive $21 .{ }^{34}$ These categories define the strategic character of the targets, including Chemical, Commercial Facilities, Communications, Critical Manufacturing, Dams, Defense Industrial Base, Emergency Services, Energy, Financial Services, Food and Agriculture, Government Facilities, Healthcare and Public Health, Information Technology, Nuclear Reactors / Materials / Waste, Transportation Systems,

February 2021.; Recorded Future. "Egregor Ransomware, Used in a String of High-Profile Attacks, Shows Connections to QakBot". 3 December 2020.; Heimdal Security. "Egregor Ransomware: Origins, Operating Mode, Recent Incidents". 13 January 2021.; McAfee. "Clop Ransomware". 1 August 2019. ; SonicWall. "A NEW VARIANT OF CLOP RANSOMWARE SURFACES". 26 October 2020.; Cynet. "THREAT REPORT: LOCKBIT RANSOMWARE". 3 June 2020.; Steve Zurier. "Payment processor used by government hit by 'Cuba' ransomware gang". SC Magazine. 19 February 2021.; Mathew J. Schwartz. "Cuba' Ransomware Gang Hits Payment Processor, Steals Data". Bank InfoSecurity. 19 February 2021.; Acronis. "DarkSide Ransomware Does Not Attack Hospitals, Schools and Governments". August 2020. ; Digital Shadows. "DarkSide: The New Ransomware Group Behind Highly Targeted Attacks". 22 September 2020. ; CrowdStrike. "BitPaymer Source Code Fork: Meet DoppelPaymer Ransomware and Dridex 2.0". 12 July 2019. ; Trend Micro. "An Overview of the DoppelPaymer Ransomware". 5 January 2021.; DarkTracer. "[BREAKING] DarkTracer discovered the new leak site of "\#Everest" \#ransomware group". https://twitter.com/darktracer_int/status/1339145675452268550; Mathew J. Schwartz. "Ransomware Newcomers Include Pay2Key, RansomEXX, Everest". Bank InfoSecurity. 2 February 2021.;CrowdStrike. "Double Trouble: Ransomware with Data Leak Extortion, Part 1". 24 September 2020.; FireEye. "Navigating the MAZE: Tactics, Techniques and Procedures Associated with MAZE Ransomware Incidents". 7 May 2020. ; Malwarebytes. "Maze: the ransomware that introduced an extra twist". 29 May 2020.; Blackberry. "MountLocker Ransomware-asa-Service Offers Double Extortion Capabilities to Affiliates". 11 December 2020.; Dissecting Malware. "Between a rock and a hard place - Exploring Mount Locker Ransomware." 23 December 2020.; Trend Micro. "Reflective Loading Runs Netwalker Fileless Ransomware". 18 May 2020.; CrowdStrike. "TECHNICAL ANALYSIS OF THE NETWALKER RANSOMWARE". 10 June 2020. ; eSentire. "The SunWalker Incident: Netwalker and SunCrypt Ransomware Double-Double Exploitation". 28 September 2020.; Blackberry. "Nefilim Ransomware and Mitigating Attacks in the COVID-19 Era". 15 June 2020.; Trend Micro. "An Analysis of the Nefilim Ransomware". 23 February 2021.; Checkpoint. "Ransomware Alert: Pay2Key". 6 November 2020. ; Cyware. "Pay2Key Ransomware's Mayhem Continues". 24 December 2020.; ANSSI. "Attacks involving the Mespinoza / Pysa Ransomware." March 2020. ; DFIR Report. "PYSA/Mespinoza Ransomware". 23 November 2020.; Kaspersky. "RansomEXX Trojan attacks Linux systems". 6 November 2020.; Trend Micro. "Expanding Range and Improving Speed: A RansomExx Approach". 6 January 2021.; Sophos. "Ragnar Locker ransomware deploys virtual machine to dodge security". 21 May 2020.; McAfee. "RagnarLocker Ransomware Threatens to Release Confidential Information". 9 June 2020. ; CyberReason. "Sodinokibi: The Crown Prince of Ransomware". 5 August 2019. ; Secureworks. "REvil/Sodinokibi Ransomware". 24 September 2019.; Tomas Meskauskas. "Egregor: Sekhmet's Cousin." Security Boulevard. 29 October 2020.; Charlie Osbourne. "As Maze retires, clients turn to Sekhmet ransomware spin-off Egregor". ZDNet. 4 November 2020.; CyWare. "SunCrypt Ransomware Takes Extortion Threats to Next Level". 5 October 2020. ; CrowdStrike. "Double Trouble: Ransomware with Data Leak Extortion, Part 2". 6 October 2020.

${ }^{33}$ Catalin Cimpanu. "Here's a list of all the ransomware gangs who will steal and leak your data if you don't pay". ZDNet. 21 April 2020.

${ }^{34}$ White House. "Presidential Policy Directive -- Critical Infrastructure Security and Resilience." 12 February 2013. 
and Water and Wastewater Systems Sectors. Some victims were identified which operate across multiple sectors, in these cases the firm was characterized by its primary line of business according to revenue in order to avoid double counting of potential access and effects.

While it has been recognized that this framework omits certain key functional enterprises that are vital to the global economy, and some limited efforts have been pursued to classify additional sectors and critical functions including, none of these designations has reached the same level of prominence and are therefore excluded from this analysis in the interests of consistency with prior surveys across the homeland security enterprise. It is acknowledged that this results in exclusion of a number of high-profile ransomware incidents from the dataset that impacted firms active in the architecture \& construction, fashion and apparel, home goods \& furnishings, hospitality, luxury goods and services, media \& entertainment, sports, and (individual) retail businesses - among others. However, there is room for robust debate to what degree many of these activities may in fact be critical to the nation in the short term time horizon of a ransomware event.

The decision to limit this analysis to encompass cases only in the United States or Europe was made based on adversary focus. While some ransomware activity has recently expanded to encompass global targeting - with a handful of potential incidents identified in the Middle East, Latin America, Asia, and Africa - criminal operators have focused far more attention on targets in more developed markets. As a result, potential strategic insights are more clearly delineated with lower probability of confounding effects due to unknown factors in individual cases treated in aggregate. Of the resulting dataset, $25.8 \%$ of cases (245) were observed in Europe, with the remainder impacting the US and Canada (704).

Initial access broker offers encompass $26 \%$ of the identified cases (243), although cases identified through ransomware extortion demands comprise the bulk of identified incidents (706). This likely results from increasing vertical integration, or at least recurring transactional stability, within continuing criminal enterprises. In a number of cases, victims first identified through initial access offers do appear to have been transactionally acquired and acted upon by the larger integrated extortion groups, based on traceable characteristics described by the broker and later observed in ransomware incidents. In such cases, the incident is classified within the dataset as a ransomware event. A large number of ransomware events however do still occur based on bulk spam and drive by exploit kit operations, delivering commodity first stage malware indiscriminately to a large number of opportunistically acquired victims.

On average 73 specific targets were identified at risk in given 30 day period. This average however obscures accelerating numbers of incidents from $4^{\text {th }}$ quarter 2020 onward, in which continued operational success and profitability of these crimes attracted larger numbers of participants in the underground market ecosystem. During this period, average targets at risk increased to over 103 victims per month; with peaks of 131 targets in September 2020, and 151 targets in November 2020. 


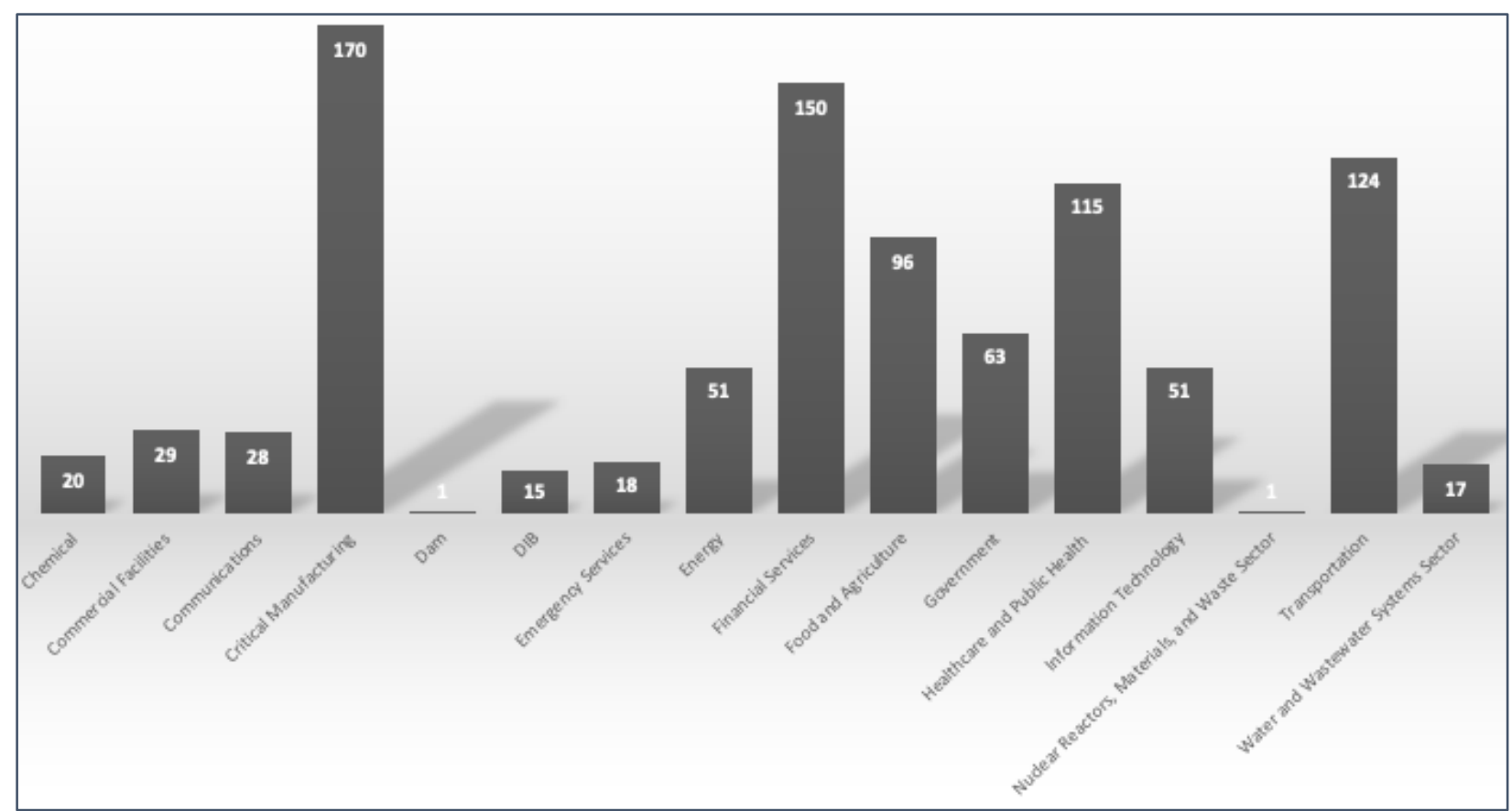

Figure 1: Incidents per critical infrastructure sector, February 2020 - February 2021

Impact to critical infrastructure sectors varied extensively, likely based on developing operator preferences as access and extortion crews obtained experience with similar victims and pursued adjacent businesses found to be both viable targets and profitable payoffs. These result in distinct trends over time within the incident history. Critical manufacturing targets represented the largest number of victims, likely as a result of target specific trends in relative information technology infrastructure age and associated lower defensive cybersecurity spend, as well as limited business continuity options creating additional pressure for payment to minimize duration of disruption to ongoing contracts with specified delivery times as part of customers" "just in time" inventory strategies or other production optimization approaches. ${ }^{35}$ Similar factors prioritizing availability against business pressures, and generally older generations of information technology investments with limited recovery options may also be seen in transportation sector incidents, which make up the third most frequently targeted sector identified in this analysis. These factors for both sectors are further aggravated by expanding functionality in newer generations of ransomware that is intended to impact industrial control systems processes, evolving from functions first pioneered in the EKANS / SNAKEHOSE family now more widely incorporated across other commodity payloads. ${ }^{36}$

\footnotetext{
${ }^{35}$ Sumer C. Aggarwal. "MRP, JIT, OPT, FMS?". Harvard Business Review. September 1985.; Uday Karmarkar. "Getting Control of Just-in-Time". Harvard Business Review. September-October 1989. ; Dragos. "Manufacturing Threat Perspective". 12 November 2020. ; Trend Micro. "The Impact of Modern Ransomware on Manufacturing Networks". 1 December 2020.

${ }^{36}$ Dragos. "EKANS Ransomware and ICS Operations". 3 February 2020. ; FireEye. "Financially Motivated Actors Are Expanding Access Into OT: Analysis of Kill Lists That Include OT Processes Used With Seven Malware Families". 15 July 2020. ; Daniel Kapellmann Zafra. "Hello From The OT Side!". Virus Bulletin Conference. Localhost. 30 September - 2 October 2020.
} 
Financial services have remained a longstanding focus of adversary action, for obvious reasons that mirror Sutton's Law. ${ }^{37}$ Yet these incidents highlight actions against the complex web of investment, payroll, escrow, accounting, legal, and tax services that are not as commonly considered within general conceptions of the sector that remain anchored on retail banking or Wall Street trading houses. The impact of such incidents, while not reaching targets identified as systemically important within regulatory constructs designed to address economic risks created by institutions "too big to fail", nonetheless may create substantial cumulative impact to populations depending on these services for matters that touch upon their daily lives.

Ransomware operators continued to target healthcare and public health aggressively during the period of analysis, despite much publicity regarding alleged declarations of voluntary restraint by some ransomware gangs fearing backlash due to the disproportionate impact of their actions during the global pandemic. The prospect of additional law enforcement pressure does not appear to have changed operator calculus here. Proposals for specific interventions by state military and intelligence services, and the declared intentions by both Australian and French governments to employ such models, likewise do not appear to have influenced ransomware operator thinking absent notable disruptive engagements. ${ }^{38}$

Ransomware operators, and the initial access brokers that supply these continuing criminal enterprises, do attempt their own characterization of strategic value. However, this is based almost entirely on financial factors associated with ability to pay high dollar value ransom demands; as well characteristics associated with scope and complexity of exploitation operations based on size of network, identified backup solutions present, and other features identified through preliminary network reconnaissance. Financial valuation is based almost entirely on cursory open source data, and determines transactional prices and attention within underground markets. While most targeted enterprises are valued variously in the tens of millions to hundreds of millions (US Dollar), a few multibillion-dollar valuations were notably observed. The unreliability of these figures precludes further analysis, although it is clear that these factors weigh heavily upon underground market participants.

It should be emphasized that this dataset certainly does not encompass the entirety of all ransomware incidents that occurred during this period. A substantial number of incidents involve only direct impact to targeted firm, for which there was no proffered access by a broker, and in which the victim may promptly negotiate and pay - therefore resulting in no pressure from the extortion operators via demand and leak sites. Such streamlined events produce few observables. However, this unknown universe of accesses may potentially be diverted for strategic campaigns by state-nexus threat actors at any time - multiplying potential

\footnotetext{
${ }^{37}$ Leonard Buder. "Willie Sutton Said It First: 'That's Where the Money Is'". New York Times. 26 August 1979.

38 JD Work. "Paging A Joint Task Force: Cyber Defense of Pandemic Medical Infrastructure". 24 March 2020. ; Helene Fouquet. "France Not Ruling Out Response to Cyber Attack on Hospital". Bloomberg. 28 November 2019. ; Australian Government, Department of Defense. "On the offensive against COVID-19 cyber criminals". 7 April 2020.
} 
victimization several times over. As a result, the dataset here should be seen as establishing a minimum floor of potential strategic impact.

Criminal ransomware operators have themselves recognized this potential. In an interview with a marketing publication run by Western cyber threat intelligence firm Record Future, one of the key actors involved in the REvil / Sodnobicki / PINCHY SPIDER enterprise stated "It is quite feasible to start a war. But it's not worth it -the consequences are not profitable." ${ }^{39}$ While this actor also made several likely aggrandized claims regarding access to military targets, almost certainly intended to influence rather than inform, it is clear the nascent kernel of strategic targeting logics are already present in successful, stable and long-running campaigns.

Taken together, a state nexus adversary seeking to leverage existing initial access brokerage and ransomware relationships for a destructive and disruptive campaign from a cold start may be presumed to offer capabilities to service approximately one hundred targets within given 30 day crisis period using solely this capacity. Nearly half of these entities may be anticipated to fall within the traditional strategic bombing target lists, encompassing transportation, energy, manufacturing, and defense industrial base objectives. Demand pull effects, patriotic nationalism, surge interest, and the influence of krysha relationships with host state services may be estimated to perhaps as much as double this within a short engagement window. The bulk of these targets may be anticipated to encompass mid-size enterprises. This represents a subset of the total constellation of an estimated 200,000 possible targets in the United States alone, accounting for a third of employment and of US Gross Domestic Product in aggregate as of 2019. ${ }^{40} \mathrm{~A}$ short, sharp cold start campaign in this model may thus be estimated to hold at risk approximately $\$ 650$ billion USD of economic value, with impact to upwards of 4.75 million employed individuals. This figure may be seen for comparative illustration as twice the federal government employment roster, and the sum of total US government discretionary spending in 2020 (not including pandemic stimulus) - although long term losses are unlikely to be realized in full value, as businesses recover from impact and restore economic activity. ${ }^{41}$

\section{Conclusion}

The prior sections have shown that while concepts of offensive cyber capabilities employment have properly evolved to emphasize the campaign as the proper unit of analysis, adversary options remain to generate substantial options to deliver effects within compressed timelines. These findings demonstrate that underground access may indeed be leveraged in cold start scenario to deliver effects that take on a strategic character.

\footnotetext{
${ }^{39}$ Recorded Future. "'I scrounged through the trash heaps... now I'm a millionaire:' An interview with REvil's Unknown". 16 March 2021.

${ }^{40}$ Ohio State University, National Center for the Middle Market. "At the Center Of Change: 2019 Annual Report." March 2020.

${ }^{41}$ Frank Konkel. "Annual Government Spending Approaches Historic Territory". 5 August 2020.
} 
However, it remains unclear at what threshold such actions will reach what is truly strategic impact. While US analysts and planners have for multiple decades recognized that sustained destructive and disruptive actions against critical infrastructure targets may generate cascading, higher order consequences more than the sum of the individual networks impacted, these theories have yet to be tested in real world fires at scale and in repeated re-strike. ${ }^{42}$ This lack of fidelity regarding the ultimate decisions in the contest of arms in and through cyberspace at the highest end of the conflict spectrum has led to a recurring bias which dismisses the potential for worst case scenario outcomes, preferring instead to assume that states can and will continue to absorb such losses as a matter of course. This also remains an untested proposition. It is equally if not more likely that states will react quite differently when faced with strategic cyber attack campaigns that exhibit substantial discontinuities with prior single target events, or even observed multiple victim uncontrolled propagation incidents where destructive effects may be downplayed as unintended results arising from misfires or improper weaponeering. ${ }^{43}$

\section{Parasitic burdens and tipping points}

A key challenge in relying upon data regarding disruptive incidents that already impact major economic targets is that such effects are essentially baked into contemporary cyber threat picture. Firms suffer from these events, struggle to reconstitute, and carry on. National economies have proven to be sufficiently resilient that they can continue to endure despite the burden placed on technology ecosystems by parasitic criminal enterprise. ${ }^{44}$ However, these events are not intended by the adversary to deliver permanent effects - they are at their core reversible in concept and execution, barring failure modes. These are also intended by both victim and actor to remain relatively less well known - at least for the subset of victims that pay promptly, and wish to minimize negative publicity.

A cold start campaign leveraging rapid capability generation options from underground marketplace accesses would likely differ from these key characteristics. While these options may be developed within the relatively short window of the crisis period, in the key weeks of escalating tensions between states over a particular dispute, it is more likely that effects delivery may be concentrated over mere days. Adversaries wishing to maximize coercive and signaling value of such operations may choose to highlight the engagements, including through various degrees of plausible or implausible deniability offered by the use of attribution fronts. And rather than intentionally reversible effects, adversary weaponeering may encompass deliberate options intended to destroy critical network equipment or operational technologies for maximum enduring damage - both to complicate individual target recovery and strain overall national resilience.

\footnotetext{
${ }^{42}$ Winn Schwartau. Information Warfare: Chaos on the Electronic Superhighway. Thunder's Mouth Press. 1994. ; Gregory J Rattray. Strategic Warfare in Cyberspace. London, England: MIT Press. 2001. ; Austin Long. "A Cyber SIOP? Operational Considerations for Strategic Offensive Cyber Planning." Journal of Cybersecurity. 3:1, 19-28. 2017.

${ }^{43}$ Work, 2020

${ }^{44}$ Formulation of the problem as one of parasite burden is owed to Cory Doctorow. "All Complex Ecosystems Have Parasites". O'Reilly Emerging Technology Conference. 16 March 2005.
} 
Concentrated, higher profile events of this nature may therefore intensify pain for the targeted sectors far beyond the current burden of continuing criminal threats. It remains unclear at what tipping point incident response consulting, managed security and business continuity services will no longer be able to effectively support victimized clients during a high number of simultaneous major events. Testimony by one of the most prominent vendors indicates that the firm already handles as many as 150 concurrent engagements for all causes - likely not all requiring fly away teams and complete network rebuild. ${ }^{45}$ This support calculus is further unbalanced by the prospective invocation of US government authorities under the Defense Production Act, as contemplated in aspirational policy proposals by government organizations such as the Cyberspace Solarium Commission, which may compel different priorities of response than envisioned under commercial contracts. ${ }^{46}$

\section{Below threshold engagements and learning adversaries}

Despite the challenges of extrapolating potential outcomes of deliberate campaigns leveraging prompt capabilities generation from underground access at strategic level, it is perhaps counterintuitive that these options should have less uncertainty for an adversary planner at the tactical level. The volume, variety and velocity of these otherwise below the threshold engagements has remained important live fire battle lab for adversary iterated learning, and has no doubt provided key insights into defender reactions, decision timeline, recovery approaches, and actual resiliency even where prospective adversaries have not been directly involved in the engagement. It is highly likely that certain states' ongoing espionage activities directed against industry cybersecurity professionals, including acknowledged compromise of prominent cyber intelligence and incident response firms, has provided substantial take from which to draw relatively accurate estimates of defensive detection and containment speed and efficacy, crisis management decisionmaking, and defender fatigue points. ${ }^{47}$ Further visibility has likely been obtained through source directed requirements leveraging relationships between hostile state services and continuing criminal enterprise operators, where key individuals involved in ransomware operations may be debriefed in order to obtain their own perspectives on specific incidents that may be of particular interest due to target sector, unique features of victim environment, or other characteristics of unique value for future planning. Even absent cooperative contact or continuing asset recruitment, hostile state intelligence services are often

\footnotetext{
${ }^{45}$ Kevin Mandia. Testimony before the U.S. House Committee on Oversight and Reform, and House Committee on Homeland Security. 26 February 2021.

${ }^{46}$ Cyberspace Solarium Commission. Final Report. March 2020.

${ }^{47}$ FireEye. "FireEye Shares Details of Recent Cyber Attack, Actions to Protect Community". 8 December 2020.; MalwareBytes. "Malwarebytes targeted by Nation State Actor implicated in SolarWinds breach. Evidence suggests abuse of privileged access to Microsoft Office 365 and Azure environments". 19 January 2021. ; Mimecast. "important update from mimecast". 12 January 2021. ; Mimecast. "Important Security Update". 26 January 2021. ; Fidelis. "Ongoing Analysis of SolarWinds Impacts". 26 January 2021. ; George Kurtz. "Testimony on Cybersecurity and Supply Chain Threats". Senate Select Committee On Intelligence. 23 February 2021. ; Qualys. "Qualys Update on Accellion FTA Security Incident". 3 March 2021.
} 
well positioned to obtain visibility into these ongoing operations through their own computer network exploitation efforts leveraged for $4^{\text {th }}$ party collection missions. ${ }^{48}$

The intelligence value alone obtained from these various accesses provides additional malicious incentives for hostile states to adopt a permissive posture towards, or even engage in clandestine sustainment of underground markets that will further iterate lessons learned from attack and defense in an ever-changing technology environment. Such relationships have been previously identified in the scope of different types of proxy problems considered within the attribution literature. ${ }^{49}$ The cultivation of high op-tempo capacities here transforms this learning activity into a unique kind of strategic latency. ${ }^{50}$

\section{Warning problems}

Like any strategic latency concern, scenarios under which hostile states leverage initial access brokerage to accelerate timelines in bootstrapped campaigns perforce reduces opportunity for intelligence warning. While understanding and defeating ongoing ransomware operations are undoubtably an intelligence priority for many friendly intelligence services (both government and private sector), the frequency of these events likely serves to dull recognition of moments when an adversary may depart from routine degradation of systems and networks for criminal profit and move towards more deliberate state objectives. Such conditioning introducing cognitive error here is somewhat unique, in that it does not emerge as in the more common failure mode from deliberate deception resulting in false alarm, but rather from routine compromises that do indeed inflict harm. Over time, collectors and analysts naturally risk becoming desensitized by the sheer volume and frequency of incidents, not merely as a problem of separating signal from noise, but from the challenge of focusing on small variance in the drumbeat of otherwise high intensity signals. ${ }^{51}$

The organizational structures built up to deal with mere crime, however widespread, also tend to differ markedly from the agencies with particular competence in addressing the exchange of fires under conflict conditions. This challenge extends beyond differing governmental agencies

\footnotetext{
48 Juan Andrés Guerrero-Saade and Costin Raiu. "Walking in your enemy's shadow: when fourth-party collection becomes attribution hell." Virus Bulletin Conference. Madrid, Spain. 4-6 October 2017.

${ }^{49}$ Jason Healey. "Beyond Attribution: Seeking National Responsibility in Cyberspace". Cyber StateCraft Initiative. Atlantic Council. 22 February 2012. ; Tim Maurer. Cyber Mercenaries: The State, Hackers, and Power. Cambridge, England: Cambridge University Press. 2018.

${ }^{50}$ Concepts here of strategic latency are owed to the excellent series of discussions hosted by Lawrence Livermore National Laboratories, the National Intelligence University, US Special Operations Command, and other partners. See Zachary Davis, Michael Nacht, Ronald Lehman [eds]. Strategic Latency and World Power. Center for Global Security Research, Lawrence Livermore National Laboratory. 2014.; Zachary Davis, Frank Gac, Michael Nacht. Strategic Latency And Warning. 2016 ; Zachary S. Davis and Michael Nacht. Strategic Latency: Red, White, and Blue. Center for Global Security Research, Lawrence Livermore National Laboratory. 2018. ; Zachary S. Davis, Frank Gac, Christopher Rager, Philip Reiner, Jennifer Snow [eds]. Strategic Latency Unleashed. Center for Global Security Research, Lawrence Livermore National Laboratory. 2021.

51 The formulation of signal and noise in intelligence warning is of course owed to Roberta Wohlstetter. Pearl Harbor: Warning and Decision. Palo Alto, CA: Stanford University Press. 1963.
} 
to also encompass private sector intelligence providers, where functional specialization in cyber crime often results in differing analytic branch or higher-level management divisions that separate these staff from others working on state-nexus threats. Internal disputes over collection priorities, and the associated recurring ad-hoc solutions in which specific core collectors are assigned in direct support of specific functions, or where analysts may even conduct their own collection, further may reinforce disconnects.

Such disconnects may be further amplified in that routine collection activities effective against continuing criminal enterprises and their transactional exchanges in the underground marketplace may well miss interactions with state services conducted through more unusual channels. Additional communications security will almost certainly be pursued by hostile military and intelligence services that are well aware of the mechanisms of collection against cyber crime targets. These hostile planners may have visibility into specific sources and methods used by friendly services by virtue of espionage providing stolen intelligence take, either as may be derived from compromised finished intelligence channels, or even from raw collects exfiltrated directly from prior intrusions against commercial security providers.

\section{Strategic (in)stability}

These dynamics highlight the destabilizing nature of pervasive vulnerability and continuing exploitation of exposed critical infrastructure below the threshold of acknowledged armed conflict. In effect, these intrusions serve in aggregate as a type of operational preparation of the environment (OPE), resulting in pre-positioned access and options to deploy destructive implants against a variety of vital networks comparable to that achieved over longer, more deliberate and covert campaigns. The problem of cold start scenario is further aggravated by the reduction of uncertainty in comparison to other incidents in which the dilemma of indistinguishability between mere espionage and destructive effects offers some possibility that a detected incident may not be intended for immediate harm. ${ }^{52}$ In the case of ransomware associated intrusion accesses, harm is highly likely manifest. It is only then the question of whether such harm is instrumental to extortion alone, and therefore potentially transient (assuming coerced payments are provided, or other response measures reverse cryptoviral effects), or if initial ransomware like tradecraft may simply provide cover for action for delivery of effects that are more difficult to recover from.

States cannot afford to ignore the potential for damage to their interests in such a scenario. The immediate impact to critical infrastructure, and the potential domestic political effects, of such an attack campaign - particularly one that rises to public attention above the routine background. In crisis, this risks potentially more escalatory reactions to attempted degradation, particularly if intended for coercive objectives, than even in the case of deliberately conducted offensive campaigns leveraging unique capabilities organic to a hostile state service. The

\footnotetext{
${ }^{52}$ Gary Brown. "Spying and Fighting in Cyberspace," Journal of National Security Law \& Policy. 2016. ; Aaron Brantly. "Aesop's Wolves: The Deceptive Appearance of Espionage and Attacks in Cyberspace." Intelligence \& National Security. 31:5, 674-85. 2016.
} 
adversary essentially risks taking ownership of the recurring pain of ordinary criminal events in such a scenario, and unleashing a response grounded as much in frustration over inadequate response to the wider global problem as in the fear of further punishment from the extant conflict dyad.

\section{Countering prospects}

Analysis of the problem of potential cold start campaigns for prompt effects identified here further appears to validate the core theory underlying the doctrine of persistent engagement. Given the years-long inability to meaningfully alter the steady recurring intrusions with disruptive and destructive effect for criminal objectives, it follows that wider deterrence against states that would leverage these access options in a time of crisis for their own objectives is a forlorn hope at best. In face of such strategic imbalances, it becomes imperative to change facts on the wire where one does not see much likelihood of success in impacting adversary decisionmaker calculus. The recognition of these conditions, and the need to actively contest adversary freedom of action to hold US and allied interests at risk, has led to the new doctrine. ${ }^{53}$

Widespread defender vulnerability, inefficiencies of coordination, and the challenges of response to the large numbers of private sector entities that need support beyond reasonable investment expectations leaves states with few options in the near term, especially when anticipating potential crisis moments. As a result, counter-cyber operations approaches may prove to be the first tool - and if not the best tool, then the best available option, that states may choose to reach for in times of need.

It is precisely these dynamics that appear to underlie reported action by US Cyber Command in advance of the 2020 US Federal elections. Concerns that ransomware associated accesses could provide a cold start advantage to deliver effects against widely vulnerable state and local networks within the time sensitive window of polling and canvassing were not insignificant factors in threat assessments of potential foreign interference. While intelligence diplomacy, backed by declarative statements, were also likely important, the US government acknowledged action in September 2020 against the Trickbot botnet infrastructure, a prominent infection vector for multiple ransomware operations. These actions followed previously unattributed counter-cyber operations that degraded transactionally related Emotet botnet operations in July 2020. These actions, and further intervention through judicially authorized takedown processes led by Microsoft, key criminal underground capacity was degraded - but more importantly, uncertainty was introduced to complicate any adversary

\footnotetext{
${ }^{53}$ Michael P. Fischerkeller, and Richard J. Harknett. "Deterrence is Not a Credible Strategy for Cyberspace." Orbis. 61: 3, 381-393. 2017. ; Paul M. Nakasone and Michael Sulmeyer. "How to Compete in Cyberspace." Foreign Affairs. September / October 2020.Michael Fischerkeller and Richard Harknett. "Persistent Engagement, Agreed Competition, Cyberspace Interaction Dynamics and Escalation." Institute for Defense Analysis. 2018 ; Michael Fischerkeller. "Fait Accompli And Persistent Engagement In Cyberspace". War on the Rocks. 24 June 2020 .; Paul M. Nakasone. "A Cyber Force for Persistent Operations". Joint Forces Quarterly. 1st Quarter 2019. ;
} 
planners' potential reliance on this infrastructure for a cold start campaign. ${ }^{54}$ The US intelligence community subsequently assessed no foreign interference attempts to alter any technical aspects of the voting process, and no substantive cyber efforts to gain access to elections infrastructure. ${ }^{55}$

\section{Outlook}

A single successful countering campaign does not eliminate the longer-term challenges posed by the multiple channels within the underground marketplace by which adversary services may rapidly generate new capabilities to deliver prompt effects against rivals. An assertive countercyber operations posture, especially pursued through "defend forward", continues to face substantial criticism both within the United States as well as among allies and partners. It remains unclear how frequently countering options may be supported by policymakers in the face of such opposition. ${ }^{56}$ Likewise, US responses will likely continue to be constrained by policymakers overly focused on uncertainty in this new domain, and who as a result are more risk averse. ${ }^{57}$

The prospect of these scenarios playing out in future conflict also forces reconsideration of the dynamics of coercion through offensive scenarios in which costs are imposed on the target immediately, with the tacit promise to conditionally halt such measures (and potentially even reverse their effects) given capitulation by the target. In this, analogies to blockade actions may be drawn. ${ }^{58}$ These analogies become particularly salient when evaluating specific actors' own thinking on the matter, which have not only followed the analogy but considered its military applications in the context of specific campaign concepts. ${ }^{59}$ Such concepts of operation address earlier challenges in works considering blockade warfare executed in and through cyberspace, which largely contemplated only the more transient effects of distributed denial of service attack, based on dynamics observed in earlier conflict in Estonia and Georgia. ${ }^{60}$ The relative utility of such operations may be enhanced through cold start scenarios of rapid capability

\footnotetext{
54 JD Work. “Poll Position: Successful counter-cyber operations secure US election." Jane's Intelligence Review. 28 January 2021.

${ }^{55}$ Office of the Director of National Intelligence. "Foreign Threats to the 2020 US Federal Elections." DECLASSIFIED. 15 March 2021.

${ }^{56}$ Jason Healey. "US Cyber Command: 'When faced with a bully...hit him harder.'" Cipher Brief. 26 January 2018. ; Jason Healey. "Triggering the New Forever War, in Cyberspace". Cipher Brief. 1 April 2018. ; Herb Lin, Max Smeets. "What Is Absent From the U.S. Cyber Command 'Vision'". Lawfare. 3 May 2018. ; Jason Healey. "The implications of persistent (and permanent) engagement in cyberspace". Journal of Cybersecurity. Volume 5 Issue 1. 2019. ; Brandon Valeriano and Benjamin Jensen. "The Myth of the Cyber Offense: The Case for Cyber". Cato Institute. 15 January 2019.; Max Smeets. "US cyber strategy of persistent engagement \& defend forward: implications for the alliance and intelligence collection". Intelligence and National Security. 35:3, 444-453. 2020.

57 Monica Kaminska. "Restraint under conditions of uncertainty: Why the United States tolerates cyberattacks". Journal of Cybersecurity. 7: 1. 2021

58 Jenny Jun. "Coercion in Cyberspace: A Model Extortion via Encryption". Working paper, Columbia University. 24 February 2021.

${ }^{59}$ RONG Hua, LV Ming-shan, WANG Xue-tong. "Applications of integrated electronic and network warfare in blockade warfare". Journal of Dalian Naval Academy. 29:6. 2006.

${ }^{60}$ Alison Lawlor Russell. Cyber Blockades. Georgetown University Press. 2014
} 
generation, where short, sharp concentrated action may serve as limited measures seen as short of the threshold of armed conflict.

Whether adversary cold start campaigns may ultimately serve to accomplish coercive objectives, or merely inflict punishment within cyclic exchanges, remains to be seen. These options have at present arisen from unique features of continuing criminal enterprise now largely dominated by Russian speaking actors. However, as is clear in observing apparent experimentation by other actors - including China and Iran nexus threats - there are substantial incentives towards cultivation of similar strategic options. Absent any real law enforcement response or other pressures, the further development of an active criminal scene through permissive neglect or tacit and clandestine support further offers little prospective downside for states that may believe that they would obtain benefit from continuing disorder. It is clear that the current international order, and the Western states that must grapple with these challenges in crisis, are still as yet ill-equipped to face these scenarios. 